\title{
FLOW ANALYSIS OF SPACE SHUTTLE FEED LINE 17-INCH DISCONNECT VALVE
}

\author{
Max Kandula* and Daniel Pearce ${ }^{* *}$ \\ Lockheed Engineering and Management Services Co., \\ Houston, Texas
}

\begin{abstract}
A steady incompressible three-dimensional viscous flow analysis has been conducted for the Space Shuttle External Tank/Orbiter propellant feed line disconnect flapper valves with upstream elbows. The full Navier-Stokes code, INS3D, is modified to handle interior obstacles. Grids are generated by SVTGD3D code. Two dimensional initial grids in the flow cross section with and without the flappers are improved by elliptic smoothing to provide better orthogonality, clustering and smoothness to the three dimensional grid. The flow solver is tested for stability and convergence in the presence of interior flappers. An under-relaxation scheme has been incorporated to improve the solution stability. Important flow characteristics such as secondary flows, recirculation, vortex and wake regions, and separated flows are observed. Computed values for forces, moments, and pressure drop are in satisfactory agreement with water flow test data covering a maximum tube Reynolds number of $3.5 \times 10^{6}$. The results will serve as a guide to improved design and enhanced testing of the disconnect.
\end{abstract}

\section{$\underline{\text { Introduction }}$}

Quick separable disconnect valves are placed in the Space Shuttle main propulsion system propellant feed lines $\left(\mathrm{LO}_{2}\right.$ and $\mathrm{LH}_{2}$ ) at the external tank to orbiter interface. Each disconnect is comprised of two bolted circular tube sections, one on the external tank (ET) side and the other on the Orbiter (ORB) side, with each section containing a rotary flapper valve that is operated by a pneumatic actuator on the orbiter side. Figure 1 shows the configuration of the $\mathrm{LO}_{2}$ disconnect. A linkage exists between the actuator and the drive and follower arms attached to the flappers. The flappers are oriented at different angles of attack to the main flow leaving the upstream elbows. One of the main design requirements is the flapper stability in the open position so that the flappers do not close during flow. Other design considerations include cavitation margin and pressure drop across the valve.

Extensive experimental data on the disconnects with simulated water flow have suggested that over a certain range of flapper angle orientations the lift forces in the direction of opening begin to decrease above a certain flow rate. Such a reduction in lift force can lead to the closing of the flappers during flight, which can be catastrophic to the mission and crew safety. A detailed fluid dynamic analysis of the flow around the flappers is therefore important for improved design and specification of stable flapper orientations.

Incompressible three-dimensional Navier-Stokes simulation of fluid flow has thus regained increased attention in recent years in view of their application to propulsion systems such as described above. Although several threedimensional codes are developed for compressible flows (e.g. Refs. 1-2), very few solution codes are presently available for the analysis of incompressible flow. The problem of pressurevelocity coupling in incompressible flow is generally handled by Poisson equation for pressure ${ }^{3}$, vorticity-velocity formulation ${ }^{4-5}$, pressure-correction equation 6 or artificial compressibility. ${ }^{7-9}$

\footnotetext{
* Principal Engineer, Member AIAA

** Senior Associate Engineer
}

This paper is declared a work of the U.S. Government and is not subject to copyright protection in the United States.
Although not time-accurate, the method of artificial compressibility generates a hyperbolic system of equations in primitive variables that can be conveniently solved for steady flows in generalized curvilinear coordinates. Kwak et al. ${ }^{9}$ have recently developed INS3D code to handle incompressible flows using this method. This code is tested for internal and external flows and in laminar and turbulent flow. However, this code in its original form does not accommodate internal obstacles when applied to internal flows. The present paper discusses the modifications made to the flow solver with application to the flow analysis of Space Shuttle quick separable disconnect valve.

\section{Analysis}

\section{Physical Assumptions}

Because of the complexity of the geometry and the flow characteristics, a number of simplifying assumptions have been made to achieve a practical solution. The major assumptions follow.

1. The flow is assumed to be steady.

2. Flow visualization during water tests have indicated that at a given flow rate, the flappers settle to a new equilibrium position about which they vibrate. In this analysis the flappers are assumed to be stationary about their equilibrium position, and any fluid-structure interaction is ignored.

3. The linkages (drive and follower arms) are removed from consideration. Although the linkages contribute to the overall pressure drop, their modeling involves prohibitively large computing time, so that their contribution to the pressure drop can be approximately evaluated by a simpler correlation.

4. Step-like roughness at the flapper surface due to linkage joints are not taken into account. Thus the flappers are taken as smooth surfaces. Similarly other walls in the system are assumed smooth. The flapper curvature and flapper angle are given primary consideration

5. The flapper edges are smoothed out, and the flapper diameters are slightly reduced from 16.3 inches to 15.1 inches to provide a reasonable gap for computation in the annular region between the flapper and the tube which is 17 " in diameter.

6. The turbulent flow is assumed to be fully developed about two tube diameters upstream of the elbow. In the test setup, a straight pipe several tube diameters long is located upstream of the elbow to allow fully developed flow near the inflow.

7. The outflow boundary is taken at about 3.5 tube diameters downstream of the orbiter flapper to allow the flow to be somewhat straightened. This assumption permits simple boundary conditions to be prescribed at the outflow.

8. The system is isothermal.

\section{Governing Equations}

The details of the artificial compressibility method and numerical algorithm for 3-D steady incompressible viscous flow are given by Kwak et al. ${ }^{9}$ Only the major features as applied to the interior obstacles will be described in detail here. 
The continuity equation is modified by the introduction of a density/pressure wave using an artificial equation of state,

$$
p^{\prime} / \rho^{\prime}=1 / \beta^{\prime},
$$

where $\beta^{\prime}$ is the compressibility parameter. As a result, the governing Reynolds-averaged Navier-Stokes equations become hyperbolic and are given in dimensionless tensor notation as

$$
\begin{aligned}
& \frac{1}{\beta} \frac{\partial p}{\partial t}+\frac{\partial u_{i}}{\partial x_{i}}=0 \\
& \frac{\partial u_{i}}{\partial t}+\frac{\partial u_{i} u_{j}}{\partial x_{j}}=-\frac{\partial p}{\partial x_{i}}+\frac{\partial \tau_{i j}}{\partial x_{j}}
\end{aligned}
$$

where

$$
\begin{aligned}
& x_{i}=\frac{x_{i}^{\prime}}{x_{r}}, \quad t=\frac{t^{\prime} u_{r}}{x_{r}}, \quad u_{i}=\frac{u_{i}^{\prime}}{u_{r}}, \\
& p=\frac{p^{\prime}-p_{r}}{\rho^{\prime} u_{r}^{2}}, \quad \tau_{i j}=\frac{\tau_{i j}^{\prime}}{\rho^{\prime} u_{r}^{2}}, \\
& v=\frac{v^{\prime}}{x_{r} u_{r}}=\frac{1}{R e}, \quad \beta=\frac{\beta^{\prime}}{\rho^{\prime} u_{r}^{2}}
\end{aligned}
$$

The primes denote dimensional quantities, and the subscript $r$ denotes reference quantities. The stress tensor is given by

$$
\tau_{i j}^{\prime}=2 v^{\prime} S_{i j}^{\prime}-R_{i j}^{\prime}
$$

where the strain rate tensor is defined by

$$
S_{i j}^{\prime}=\frac{1}{2}\left(\frac{\partial u_{i}^{\prime}}{\partial x_{j}^{\prime}}+\frac{\partial u_{j}^{\prime}}{\partial x_{i}^{\prime}}\right)
$$

The Reynolds stresses are evaluated via eddy viscosity model using a constitutive equation for the mixing length, and are given by

$$
R_{i j}^{\prime}=\frac{1}{3} R_{k k}^{\prime} \delta_{i j}-2 v_{t}^{\prime} S_{i j}^{\prime}
$$

\section{Difference Equation Formulation of INS3D}

In the INS3D code, the physical coordinates are transformed into generalized curvilinear coordinates:

$$
\tau=t, \quad \xi_{i}=\xi_{i}\left(x_{i}, t\right)
$$

where $\xi_{i}=\xi, \eta$, or $\zeta$, and $x_{i}=x, y$, or $z$ for $i=1,2$, or 3 , respectively. The governing equations are then transformed to computational space, with the Jacobian of the transformation given by

$$
J=\operatorname{det}\left[\frac{\partial \xi_{i}}{\partial x_{i}}\right]
$$

Implicit approximate factorization scheme ${ }^{10}$ is employed. Although both fully-implicit (Euler implicit, first order timeaccurate) and trapezoidal time differencing (second order time- accurate) are considered, only Euler Implicit scheme has been investigated in this work. With the smoothing terms included, the approximate factored form of the governing difference equations for fully implicit scheme in delta form become

$$
L_{\xi} L_{\eta} L_{\zeta} \Delta Q^{n+1}=F^{n}
$$

where

$$
\begin{aligned}
& L_{\xi_{i}}=\left[I+\frac{\Delta \tau}{2} J^{n+1} \delta_{\xi_{i}}\left(\mathrm{~A}_{i}^{n}-T_{i}\right)-\varepsilon_{i} \delta_{\xi_{i}}^{(2)}\right] \\
& F^{n}=-\Delta \tau J^{n+1}\left(\delta_{\xi} E_{1}^{n}+\delta_{\eta} E_{2}^{n}+\delta_{\zeta} E_{3}^{n}\right) \\
& +\Delta \tau J^{n+1}\left(\delta_{\xi} T_{1}+\delta_{\eta} T_{2}+\delta_{\zeta} T_{3}\right) Q^{n} \\
& \text { - }\left[I-\left(J^{n+1} / J^{n}\right)\right] Q^{n} \\
& -\varepsilon_{e}\left[\delta_{\xi}^{(4)}+\delta_{\eta}^{(4)}+\delta_{\zeta}^{(4)}\right] Q^{n} \\
& T_{i}=\frac{v}{J} I_{m}\left(\nabla \xi_{i} \cdot \nabla \xi_{i}\right) \delta \xi_{i} \quad \text { (orthogonal grid) } \\
& I_{m}=\left[\begin{array}{llll}
0 & 0 & 0 & 0 \\
0 & 1 & 0 & 0 \\
0 & 0 & 1 & 0 \\
0 & 0 & 0 & 1
\end{array}\right]
\end{aligned}
$$

In the above equations, $\delta_{\xi_{i}}, \delta_{\xi_{i}}^{(2)}, \delta_{\xi_{i}}^{(4)}$ are the central differences, and $\varepsilon_{i}$ and $\varepsilon_{e}$ are the implicit and explicit smoothing coefficients, respectively. The solution vector $Q$, the flux vector $E_{i}$, and the coefficient matrix of the local linearizations $A_{i}$ are defined by

$$
Q=\left[\begin{array}{c}
p \\
u \\
v \\
w
\end{array}\right], E_{i}=\frac{1}{J}\left[\begin{array}{c}
\beta U_{i}+L_{0}(p-\beta) \\
u U_{i}+L_{1} p \\
v U_{i}+L_{2} p \\
w U_{i}+L_{3} p
\end{array}\right]
$$

$A_{i}=\frac{1}{J}\left[\begin{array}{cccc}L_{0} & \left(L_{1} \beta\right) & \left(L_{2} \beta\right) & \left(L_{3} \beta\right) \\ L_{1} & \left(U_{i}+L_{1} u\right) & L_{2} u & L_{3} u \\ L_{2} & L_{1} \mathrm{v} & \left(U_{i}+L_{2} \mathrm{v}\right) & L_{3} \mathrm{v} \\ L_{3} & L_{1} w & L_{2} w & \left(U_{i}+L_{3} w\right)\end{array}\right]$

with the contravariant velocities $U_{i}$ given as

$$
U_{i}=L_{0}+L_{1} u+L_{2} \mathrm{v}+L_{3} w \quad,
$$

where the metrics of the transformation are

$$
\begin{aligned}
& L_{0}=\left(\xi_{i}\right)_{t}, L_{1}=\left(\xi_{i}\right)_{x}, L_{2}=\left(\xi_{i}\right)_{y}, \\
& L_{3}=\left(\xi_{i}\right)_{z}
\end{aligned}
$$

The numerical scheme of Eq. (7) is accurate to $\mathrm{O}\left(\Delta x^{2}\right)$ and $O(\Delta \tau)$. The above equations reduce to a block tridiagonal matrix of the form 


$$
A_{i} \Delta Q_{i-1}^{n+1}+B_{i} \Delta Q_{i}^{n+1}+C_{i} \Delta Q_{i+1}^{n+1}=D_{i}^{n}
$$

in each spatial direction, and are easily solved for $\Delta Q$ at $n+1$ iteration using successive sweeps, where

$$
\Delta Q^{n+1}=Q^{n+1}-Q^{n}
$$

\section{Modifications to INS3D}

In its original form, the INS3D code does not allow any interior obstacles for internal flow. Therefore, the code is modified to handle interior obstacles of arbitrary geometry and tested for solution stability, convergence and accuracy. This marks an important contribution of the present report. Following are the major modifications made.

1) The grid region corresponding to the interior obstacles is blanked out. Thus a so called porosity method is used for treating the flappers in the computational grid.

2) The coefficients of the block tridiagonal matrix corresponding to the blanked grid points are set such that in Eq. (9a)

$$
A_{i}=0, B_{i}=1, C_{i}=0, D_{i}=0,
$$

so that the solution at those points is not carried out.

3) Second order explicit smoothing is considered near the surface of the internal obstacles.

4) Appropriate explicit boundary conditions are imposed on the exterior of these obstacles.

5) An under-relaxation scheme has been implemented to provide enhanced stability and accuracy of the flow solution.

\section{Flow Solution}

The adjustable parameters in the solution include the compressibility parameter $\beta$, the smoothing coefficients $\varepsilon_{i}$ and $\varepsilon_{o}$, and the time step $\Delta \tau$. The stability, convergence, and accuracy of the solution is known to depend strongly on these parameters, in addition to the quality of the given grid. In general the choice of these parameters depends on the grid configuration and Reynolds number, and no definitive rules for setting their range currently exist. The choice is one of trial and error procedure. Even in laminar flow at low $R e$, the selection of these parameters is not well developed. The situation becomes more difficult at high Reynolds number and for complex geometry with interior obstacles. The present section discusses some of the guidelines reported in Ref. 9, and our observation in dealing with the present geometry.

Compressibility Parameter. A criterion for the limits of $\beta$ are given in Ref. 9 for 1-dimensional flow. The lower bound for $\beta$ is decided by the fact that the pressure wave propagates much faster than the spreading of vorticity, and is given by

$$
\beta \gg\left[1+\frac{4}{R e}\left(x_{r} / x_{\delta}\right)^{2}\left(x_{L} / x_{r}\right)\right]^{2}-1
$$

where $x_{\delta}$ and $x_{L}$ are the characteristic lengths that the vorticity and the pressure waves have to propagate during a given time span.
The upper bound of $\beta$ is given by the error associated with the approximate factorization, and is expressed as

$$
\beta \Delta \tau<O(1)
$$

For a given geometry and grid size, an optimum range of $\beta$ is seen to ensure improved stability and convergence. ${ }^{7,9}$ In order to obtain convergence in a reasonable number of iterations, and to provide a consistent selection of $\beta$, a value of

$$
\beta=C / \Delta \tau
$$

is used in the present investigation, where $C=1.0$

Time Step. The Alternating Direction Implicit (ADI) method is generally known to be inherently unstable for three dimensions. ${ }^{11}$ Consequently the choice of time step is important to ensure stability of the solution. The local time step for stability based on the explicit scheme provides a guide and is given by the acoustic (CFL condition) and diffusion limitations, ${ }^{12}$

$$
\Delta \tau=\min \left[\frac{\Delta S}{|\vec{v} \cdot \vec{l}|+c+\frac{\alpha \mu_{t o t}}{\Delta S}}\right]
$$

where $\vec{l}$ is the unit vector along the curvilinear arc distance, $\Delta S$. The quantity $\mu_{\text {tot }}$ is the total viscosity given by

$$
\mu_{t o t}=\mu+\mu_{t u r b}
$$

and $\alpha$ is the inverse of the diffusion number and is of the order of 4 (Ref. 13). The sound speed, $c$, is given by

$$
c=\sqrt{u^{2}+\beta}
$$

For problems under investigation, $u=O(1)$, and thus $c$ $\gg \mid \vec{V} \cdot \vec{l}$ I. Furthermore, for high Reynolds number flow applications, the third term in the denominator is much smaller than $c$. As a result, for high $R e$ the stability is governed primarily on the compressibility parameter, $\beta$.

Smoothing Coefficients. Numerical dissipation or smoothing terms are added to smooth high frequency oscillations associated with central differencing. A certain relation between $\varepsilon_{e}$ and $\varepsilon_{i}$ exists that maintains the stability of the solution. Based on a linear stability analysis for onedimensional flow and numerical experimentation, a relation

$$
\varepsilon_{i}=3 \varepsilon_{e}
$$

has been suggested in INS3D, and is employed in this analysis. The value of $\varepsilon_{e}$ is scaled here with $\Delta \tau$ such that

$$
\varepsilon_{e}=\Delta \tau / 2
$$

to maintain consistent definition of $\varepsilon_{e} \cdot{ }^{14}$

Initial and Boundary Conditions. For the initial condition, the streamwise velocity distribution is taken to be the same as that at inflow. The pressure is taken to be 1.0 throughout.

No slip boundary condition is used on all solid surfaces including the internal obstacles. The pressure gradient is set to 
zero on the walls, i.e.,

$$
\frac{\partial p}{\partial n}=0
$$

At the inflow, static presssure of 1.0 is specified and a power-law type turbulent flow velocity profile for fully developed conditions is employed. All the flow variables are linearly extrapolated at the outflow boundary. A correction for the streamwise velocity at time level $n$ is applied based on mass weighted extrapolation as ${ }^{9}$

$$
\left(u^{n}\right)_{o u t}=\frac{\dot{m}_{i n}}{\dot{m}_{o u t}}\left(u^{n}\right)_{o u t, e}
$$

where the subscript $e$ denotes extrapolated value. In Eq. (18), $\dot{m}_{i n}$ and $\dot{m}_{\text {out }}$ are the mass fluxes at the inflow and outflow, respectively, with the mass flux defined by

$$
\dot{m}=\int_{A} \vec{V} \cdot d \vec{A}
$$

All boundary conditions are treated explicitly, for the sake of convenience.

Turbulence Model. The nature of turbulent flow in the present geometry is complicated by the upstream bends and internal obstacles. While the well-known Baldwin-Lomax model $^{15}$ is widely used for external flow, its application to the present case may not be appropriate since the location of maximum moment of vorticity, taken as the turbulence length scale, is not well defined for internal flows. On the other hand, a higher order $k-\varepsilon$ model coded in INS3D (see Refs. 16-17) requires additional computing time, and may slow down the convergence if proper boundary conditions for $k$ and $\varepsilon$ are not specified or available for a complex geometry such as under consideration. Therefore a simple and crude turbulence model is coded initially based on Prandtl mixing length theory for turbulent shear flow in pipes. The turbulent viscosity is computed based on the assumption of local fully-developed pipe flow conditions. Thus we have ${ }^{18}$

$$
\begin{aligned}
\frac{v_{t}^{\prime}}{u^{*} R_{L}} & =k^{2}\left(1-\mathrm{e}^{-k y^{+}}\right)\left(1-y^{+}\right)^{1 / 2}, y^{+} \leq 0.8 \\
& =\frac{v_{t}^{\prime}}{u^{*} R_{L}} \quad \text { @ } y^{+}=0.8, y^{+}>0.8
\end{aligned}
$$

where

$$
y^{+}=y^{\prime} u^{*} / v^{\prime}, \quad u^{*}=\sqrt{\tau_{w}^{\prime} / \rho^{\prime}}
$$

In Eq. (20), $k$ is the mixing length constant of $0.4, y^{\prime}$ is the distance from the wall, and $\tau_{w}^{\prime}$ is the wall shear stress. The length scale, $R_{L}$, is taken as the radius of the local flow cross section normal to the stream.

Under-Relaxation. The boundary conditions at inflow and outflow, as discussed earlier, are more appropriate for an elliptic problem Thus care needs to be exercised in applying the boundary conditions rather gradually during time-iterations for promoting solution stability. Such a treatment has become necessary for high Reynolds number flows with interior obstacles . Some kind of relaxation is therefore considered to preserve the stability of the solution, as suggested in Ref. 19.

In the present study, both boundary and global underrelaxation have been found to be useful, and are conveniently implemented as

$$
Q_{i}^{n+1}=Q_{i}^{n}+\alpha_{r} \Delta Q_{i}^{n+1}
$$

where $\alpha_{r}$ is an under-relaxation factor corresponding to the pressure and the three velocity components.

The following values of $\alpha_{r}$ have been chosen in the present problem for $p$ and $u_{i}(i=1,2$ and 3$)$ :

$$
\begin{aligned}
& \alpha_{r}=(0.01,0.5,0.5,0.5), \quad J=2 \\
& \alpha_{r}=(0.05,0.5,0.5,0.5), \quad J=J M A X-1, J M A X-2 \\
& \alpha_{r}=(0.8,0.5,0.5,0.5), \quad J=2<J<J M A X-2
\end{aligned}
$$

where $J=1$ and $J=J M A X$ represent the inflow and outflow respectively.

\section{Computational Grid}

Three dimensional grids of the $\mathrm{LO}_{2}$ disconnect are generated by the SVTGD3D code developed by Soni et al. ${ }^{20-}$ 21 This code is especially suitable for internal flow with interior obstacles which are handled by an automatic patching technique. Initial algebraic grids are generated by transfinite interpolation method, and are improved by elliptic smoothing from the solution of the Poisson equation. ${ }^{22}$

A coarse grid of $54 \times 21 \times 25$ is used for development on a local VAX-8650 computer so that stability, convergence and accuracy of the solution are studied. A portion of coarse grid development was done on the Cray X-MP and Cray-2 supercomputers at NASA Marshall and NASA Ames, respectively, through long distance communication lines from NASA Johnson. Details of the flow field are observed qualitatively using a fine grid of $102 \times 35 \times 39$ run on Cray-2 at NASA Ames.

The computational grid of the $\mathrm{LO}_{2}$ disconnect for the coarse grid is shown in Figure 2. Initial 2-D H-type grids are generated in the flow cross section with and without the flappers. These grids are then smoothed by the elliptic solver using appropriate forcing coefficients and an iterative process until the grid is satisfactory in terms of orthogonality, smoothness and clustering. Grid is clustered at all solid boundaries, flapper leading and trailing edges and in the wake regions, where flow variations are expected to be large. Hyperbolic tangent functions are used for grid stretching. In regions containing the flappers, cubic Bezier curves are also specified to provide better orthogonality. The 2-D smoothed grids are later input to $3-D$ volume patching process, and global smoothing is again accomplished in three dimensions.

\section{$\underline{\text { Results }}$}

\section{Convergence History}

The convergence of the solution is measured by the RMS values of $\Delta Q$, denoted by RMSDQ. The accuracy of the solution is monitored by the quantity RMSDIV which is the RMS value of the divergence of the velocity field. Also the maximum value of $\triangle Q$, DQMAX, is checked to insure stability of the solution. 
Figure 3 presents the convergence history of the coarse grid $(54 \times 21 \times 25)$ at a full power $R e$ of $3.52 \times 10^{6}$. In these calculations, a time step of 0.002 and a $\beta$ of 500 are employed. The corresponding CFL number is about 140 . It is seen that both RMSDQ and RMSDIV converge up to about 800 iterations.

The predicted time history of pressures at the center of the ET and Orbiter Flappers is displayed in Figs. 4a and 4b, respectively. Pressures at both top and bottom surfaces are indicated. The pressure values show initial transients followed by a minimum before establishing a trend toward steady state.

Figure 5 shows the time-wise variation of the pitching moments for the ET and the Orbiter flapper. The results indicate that the pitching moments on both the flappers approach a nearly steady state condition. The ET flapper shows a positive pitching moment (clockwise), and the Orbiter flapper a negative moment (anti-clockwise). This result implies that both flappers tend to move in the open direction, and are therefore considered stable.

\section{Flowfield}

The flow solution is visualized using the PLOT3D program. ${ }^{23}$ Figure 6a presents the $x-z$ view of the mean velocity vectors in the symmetry plane at all streamwise locations. In the flow through the elbow, centrifugal forces act at right angles to the main flow and generate secondary flow. At the outer radius of the elbow, the flow decelerates initially due to an adverse pressure gradient, and then begins to accelerate downstream due to a favorable pressure gradient. The situation is just the opposite near the inner radius of the bend. The point at which the velocity has its peak is shifted to the outside. Also shown are a leading edge separation region on the ET flapper, a wake region in between the flappers, and a developing turbulent wake past the Orbiter flapper. Further downstream of the orbiter flapper the flow tends to be fully developed.

Secondary flow, recirculation, and tip vortices are seen in Figure $6 \mathrm{~b}$ and $6 \mathrm{c}$ where velocity vectors in the flow cross section containing the midplane of the flappers are plotted. The results also suggest that the grid needs to be refined in the corner regions at the tube boundary.

Pressure contours in the $x-z$ plane of symmetry are displayed in Figure 7a. This result shows the low and high pressure regions in the elbow, leading edge stagnation regions, and the wake region. Pressure contours in the flow cross section containing the midplane of the ET and Orbiter flappers are demonstrated in Figures $7 \mathrm{~b}$ and $7 \mathrm{c}$ respectively. The wiggles over the tube boundary indicates that the grid needs to be improved in that region so that the wall boundary conditions are more accurately satisfied.

A fine grid of $102 \times 35 \times 39$ is generated based on coarse grid experience, and is run on Cray- 2 and Cray X-MP machines. Efforts are underway to improve accuracy and increase convergence to steady state. The fine grid solution is qualitatively similar to the coarse grid result, but provides a more detailed flow resolution especially near the flappers.

Typical computing speeds are $2.8 \times 10^{-3} \mathrm{~s}, 1.1 \times 10^{-4} \mathrm{~s}$, and $1.5 \times 10^{-4} \mathrm{~s}$ per mesh point per iteration on VAX-8650, Cray X-MP, and Cray- 2 respectively.

\section{Comparison of Water Test Data}

The accuracy of the predicted steady state hydrodynamic loads from the coarse grid is tested by a comparison with available water test data obtained at Wyle Laboratories in California. Rockwell International, Downey, the prime contractor for the Shuttle, subcontracted the design and development of the disconnect to Parker-Hannifin, Irvine, which monitored these tests. In the water tests ${ }^{24}$, water at a pressure of $95 \mathrm{psia}$ and a temperature of $65 \pm 15^{\circ} \mathrm{F}$ is used. A video picture examination of the flappers during flow suggested that the flappers settle to a new orientation during flow about which they vibrate. The incremental angle due to settling increases relatively rapidly with the flow rate up to about the minimum power level (MPL), and varies only gradually thereafter up to full power level (FPL). At full power, the ET and orbiter angles become $5.5^{\circ}$ and $-8.5^{\circ}$, respectively, whereas their initial (no flow) angles are $4.5^{\circ}$ and $-3.5^{\circ}$, respectively. In the present grid the full power flow angles are used.

In the test, loads are measured at two stops on each flapper, with the stops located at about 1.57" from the flapper center. The reaction force measured at these stops yield the pitching moment of the flapper. At no flow, there exists a load on the flapper due to the linkages. This load is given by the effective stop load at no flow, which is added to the predicted stop load from CFD analysis. Thus the total stop load is the sum of the preload (stop load at no flow) and the hydrodynamic load.

Figure 8a shows a comparison of the predicted values and test data of the stop loads on the ET flappers as a function of the dynamic pressure. At a given flow rate, the data show oscillations of load about an equilibrium value. These oscillations are observed to be due to flapper vibration during flow about a mean position. Therefore the present predictions correspond to the average value of the data band at a given flow rate. The analysis is seen to predict the average stop load data satisfactorily over the flow rate range considered.

The comparisons of stop loads on the orbiter flapper are displayed in Fig. 8b. The analysis predicts the data well in the low flow rate range, but somewhat underpredicts the data in the higher flow rate range. The flattening of the data is found to be due to the fact that at the larger flow rate the orbiter flapper contacts the drive arm as indicated by evidence marks in a post-test examination.

Figure 9 depicts the history of computed drag force on ET and Orbiter flappers. A periodic-type drag force is noted at large time, indicating the existence of unsteady wake and vortex shedding past the trailing edges of the flappers. These unsteady effects cannot be accurately modeled by the INS3D code. No measurements of flapper drag are available at present.

Pressure drop due to flappers/linkages is measured 24 by taking differential pressure data about 15 feet upstream of the ET flapper position and 14 feet downstream of the Orbiter flapper position. The measurements are taken with and without flappers/linkages and allow pressure recovery. In the analysis, pressure drop between inflow and outflow are predicted with and without the flappers. The pressure drop due to the flappers at full power $R e=3.52 \times 10^{6}$ is estimated at about 3.1 psi. This result compares to the test data of about $4.1 \mathrm{psi}$. The underprediction of pressure drop is attributed to factors including the neglect of the drive/follower arms, the assumption of smooth walls, the coarseness of the grid, and the crudeness of the turbulence model.

A more detailed study of the stability margins of the flappers using various flapper angle combinations is in progress. Preliminary results show that the computed stop load dependence on flapper angles is in qualitative agreement with test data. 


\section{Conclusions}

The INS3D code is modified to handle interior obstacles that are present in the three-dimensional steady viscous incompressible flow analysis of Space Shuttle disconnect flapper valves at high Reynolds numbers. The stability, convergence, and accuracy of the code using a coarse grid are tested by a comparison with available water test data for hydrodynamic loads on the flappers. Details of the complex flow field, especially around the flappers, are resolved with the use of a finer grid. While the preliminary results from the code are promising, the convergence of the code needs to be accelerated while maintaining the solution stability. More effort is required in the application of a higher order turbulence model. Areas of the computational grid are identified for further refinement.

\section{Acknowledgement}

This work was supported by NASA Johnson Space Center under Contract NAS9-17900, monitored by P. E. Cota, Jr. and B. J. Rosenbaum of the Propulsion and Power Division. The authors thank Dr. D. Kwak and Dr. U. Kaul of NASA Ames Research Center for their helpful discussion and suggestions in the development of this program. Thanks are due to F. W. Martin Jr. of the Advanced Program Office at JSC for his many useful discussions and his help in the IRIS graphics support and Cray-2 communications. The National Aerodynamic Simulation Facility (NAS) at NASA Ames Research Center allocated Cray-2 computing time. NASA Marshall Space Flight Center permitted access to the Cray XMP facility. Lockheed personnel, G. W. Adams, Jr. and S. A. Trudan, provided help in the preparation of this report.

\section{References}

${ }^{1}$ Pulliam, T.H. and Steger, J.L., "Implicit FiniteDifference Simulations of Three-Dimensional Compressible Flow," AIAA Journal, Vol. 18, Feb. 1980, pp. 159-167.

${ }^{2}$ Shang, J. S., Buning, P.G., Hankey, W. L., and Wirth, M. C., "Performance of a Vectorized ThreeDimensional Navier-Stokes Code on the CRAY-1 Computer," AIAA Journal, Vol. 18, Sept. 1980, pp. 1073-1079.

${ }^{3}$ Harlow, F.H., and Welch, J.E.," Numerical Calculation of Time Dependent Viscous Incompressible Flow with Free Surface," Physics of Fluids, Vol. 8, Dec. 1965, pp. $2182-$ 2189.

${ }^{4}$ Dennis, S.C.R., Ingham, D. B., and Cook, R. N., "Finite Difference Methods for Calculating Steady Incompressible Flows in Three-Dimensions," Journal of Computational Physics, Vol. 33, 1979, pp. 325-339.

${ }^{5}$ Osswald, G.A., Ghia, K.N., and Ghia, U., "A Direct Algorithm for Solution of Incompressible Three-Dimensional Unsteady Navier-Stokes Equations," AIAA Paper 87-1139, Proceedings of AIAA 8th Computational Fluid Dynamics Conference, Honolulu, Hawaii, June 9-11, 1987.

6Patankar, S. V., and Spalding, D.B., "A Calculation Procedure for Heat, Mass and Momentum Transfer in ThreeDimensional Parabolic Flows, "International Joumal of Heat Mass Transfer, Vol. 15, 1972, pp. 1787-1806.

${ }^{7}$ Chorin, A.J., "A Numerical Method for Solving Incompressible Viscous Flow Problems," Journal of Computational Physics, Vol. 2, 1967, pp 12-26.

${ }^{8}$ Choi, D., and Merkle, C. L., "Application of Time Iterative Schemes to Incompressible Flow," AIAA Journal, Vol. 23, No. 10, October 1985, pp. 1518-1524.
${ }^{9}$ Kwak, D., Chang, J.L.C., Shanks, S.P. and Chakravarthy, S.R., "A Three-Dimensional Incompressible Navier-Stokes Flow Solver Using Primitive Variables," AIAA Journal, Vol. 24, No. 3, March 1986, pp. 390-396.

${ }^{10}$ Beam, R.M., and Warming, R.F., "An Implicit FiniteDifference Algorithm for Hyperbolic Systems in Conservation-Law form," Journal of Computational Physics, Vol. 22., Sept. 1976, pp. 87-110.

${ }^{11}$ Anderson, D.A., Tannehill, J.C., and Pletcher, R.H., Computational Fluid Mechanics and Heat Transfer, Hemisphere Publishing Co., New York, 1984.

${ }^{12}$ Richtmyer, R.D., and Morton, K.W., Difference Methods for Initial Value Problems, Wiley, New York, 1967.

${ }^{13}$ Davis, R.L., Ni Ron-Ho, and Carter, J.E., "Cascade Viscous Flow Analysis Using Navier-Stokes Equations," J. Propulsion, Vol. 3, No. 5, Sept. - Oct. 1987, pp. 406-414.

${ }^{14}$ Steger, J.L., and Kutler, P., "Implicit FiniteDifference Procedures for the Computation of Vortex Wakes," AIAA Journal, Vol. 15, No. 4, April 1977, pp. 581590 .

${ }^{15}$ Baldwin, B.S., and Lomax, H., "Thin Layer Approximation and Algebraic Model for Separated Turbulent Flows," AIAA Paper 78-257, Huntsville, Alabama, Jan. 1978.

${ }^{16} \mathrm{Kaul}$, U., and Kwak, D., "Computations of Internal Turbulent Flow with Large Separated and Secondary Flow Regions," AIAA Paper 85-1687, AIAA 18th Fluid Dynamics and Plasma Dynamics and Lasers Conference, Cincinnati, Ohio, July 16-18, 1985.

${ }^{17}$ Kaul, U.K., "An Implicit Finite-Difference Code for a Two-Equation Turbulence Model for Three Dimensional Flows," NASA Technical Memorandum 86752, NASA Ames Research Center, June 1985.

${ }^{18}$ Chang, J.L.C., Kwak, D., Dao, S.C. and Rosen, R., "A Three-Dimensional Incompressible Flow Simulation Method and its Application to the Space Shuttle Main Engine, Part II-Turbulent Flow, AIAA-85-1670, AIAA 18th Fluid Dynamics and Plasma Dynamics and Lasers Conference, Cincinnati, Ohio, July 16-18, 1985.

${ }^{19}$ Patankar, S.V., Numerical Heat Transfer and Fluid Flow, Hemisphere Publishing Co., New York, 1980.

${ }^{20}$ Soni, B.K., "Two- and Three-Dimensional Grid Generation for Internal Flow Applications of Computational Fluid Dynamics," AlAA Paper 85-1526, Proceedings of AIAA 7 th Computational Fluid Dynamics Conference, Cincinnati, Ohio, July 15-17, 1985.

${ }^{21}$ Soni, B.K., McClure, M.D., and Heikkinen, B.D., User's Guide for SVTGD3D, Sverdrup Technology Inc., Prepared for Arnold Engineering Development Center, Arnold Air Force Station, Tennessee, September 25, 1986.

${ }^{22}$ Thompson, J., Warsi, Z. U. A., and Mastin, C.W., Numerical Grid Generation - Foundations and Applications, Elsevier Science Publishing Co., Inc., New York, 1985.

23Buning, P., PLOT3D Graphics Program, NASA Ames Research Center, 1986.

${ }^{24}$ Jones, D. L., "17-Inch Disconnect Preload Margin Development Test Program," Rockwell International, Doc. \#MCR 10473 R5, Downey, CA., October 25, 1985. 


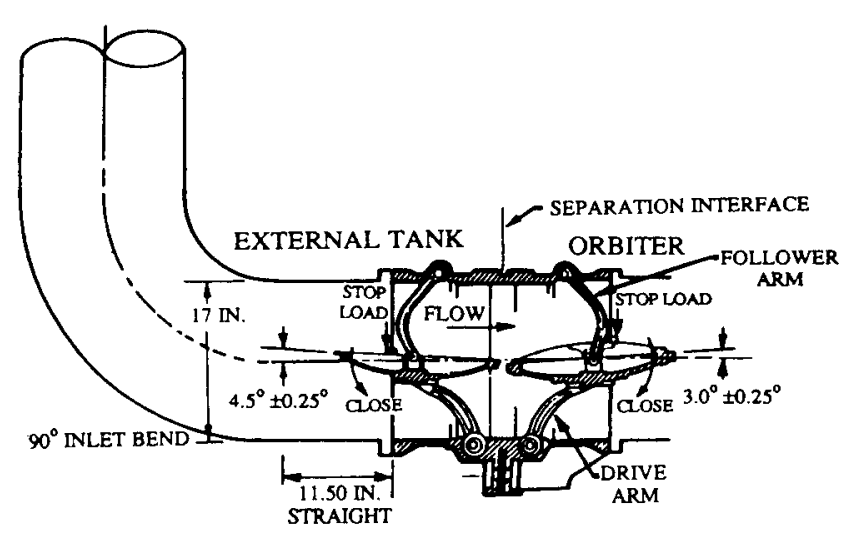

Fig. 1 Configuration of $\mathrm{LO}_{2}$ disconnect valve.

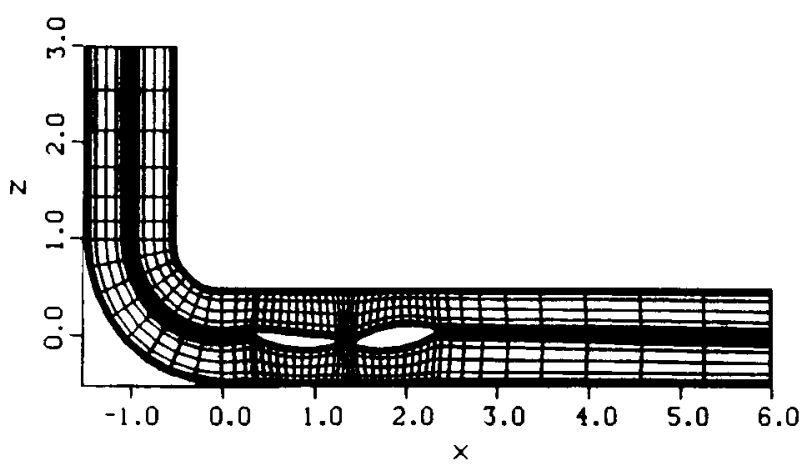

Fig. 2a $x-z$ view of the grid at the plane of symmetry.

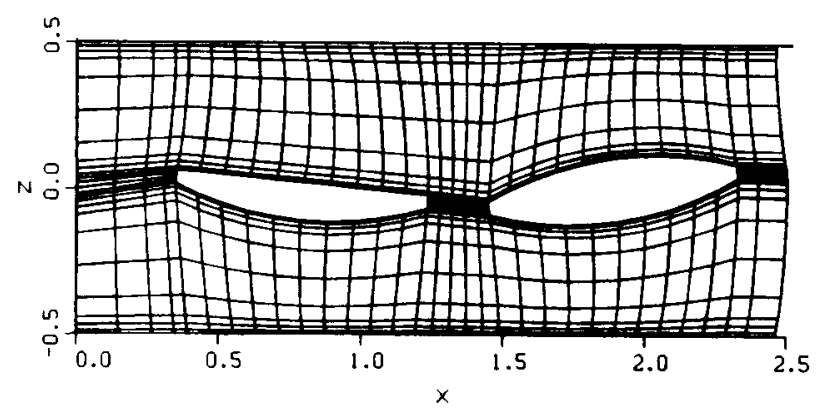

Fig. $2 b \quad x-z$ view of the grid near the flappers.

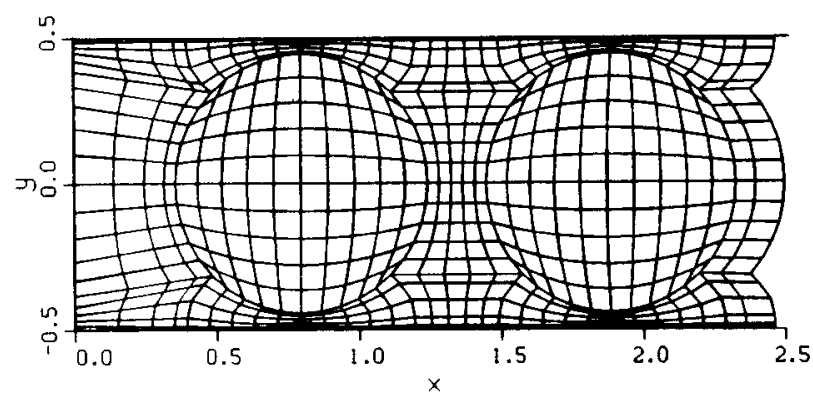

Fig. $2 c x-y$ view of the grid in the midplane of the flappers.

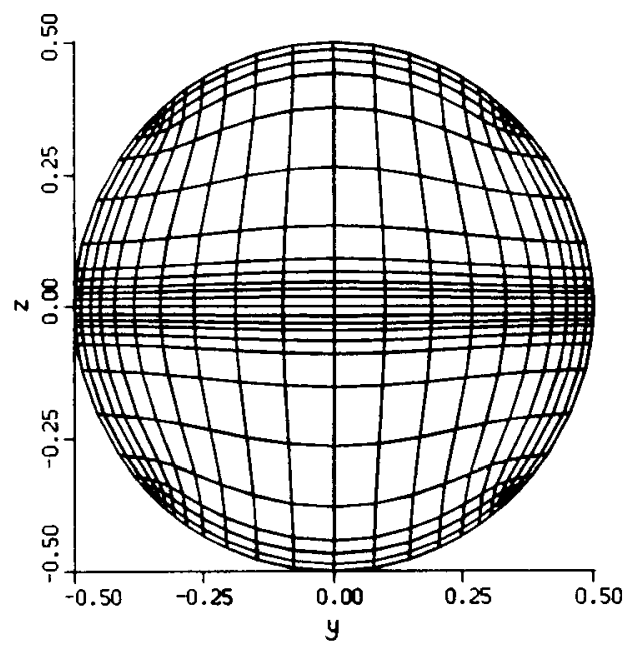

Fig. 2d Cross-sectional view of the grid at inflow and outflow.

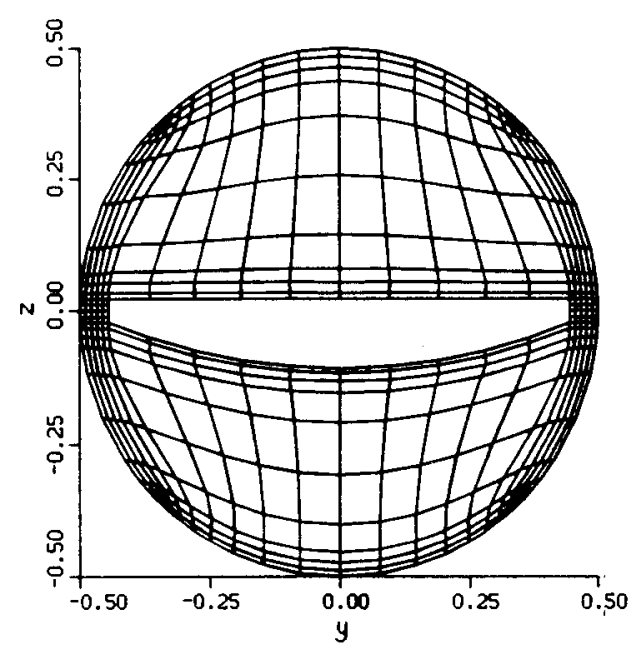

Fig. 2e Cross-sectional view of the grid at the center of the ET flapper.

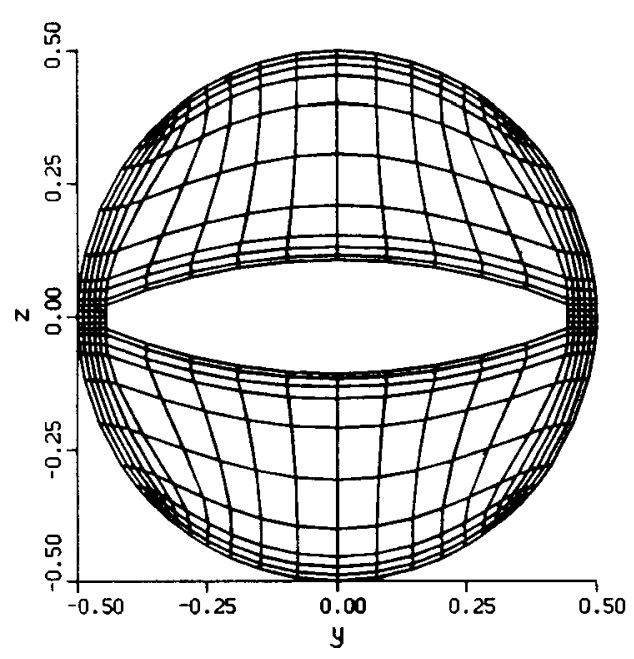

Fig. $2 \mathrm{f}$ Cross-sectional view of the grid at the center of the Orbiter flapper. 


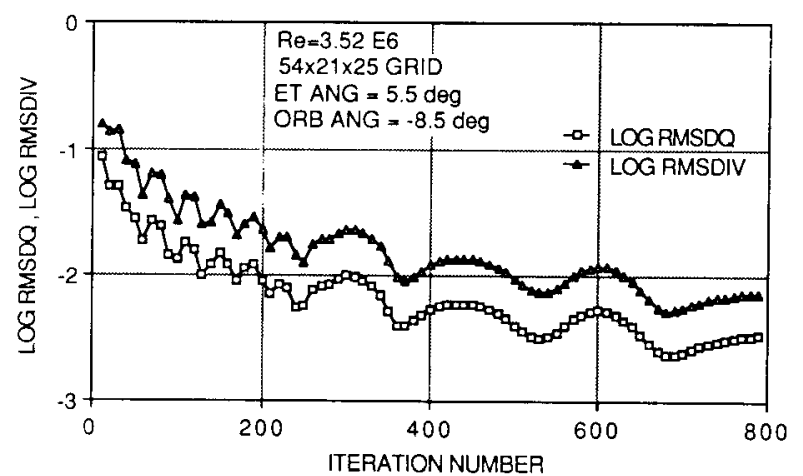

Fig. 3 Convergence history.

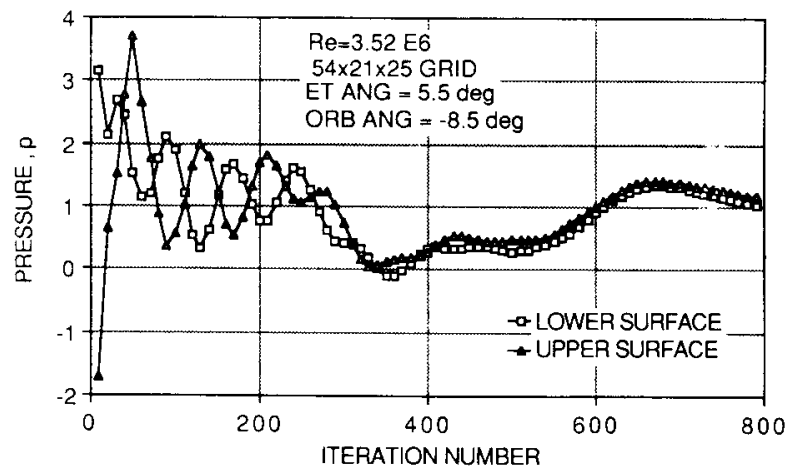

Fig. 4a Pressure history at the center of the ET flapper.

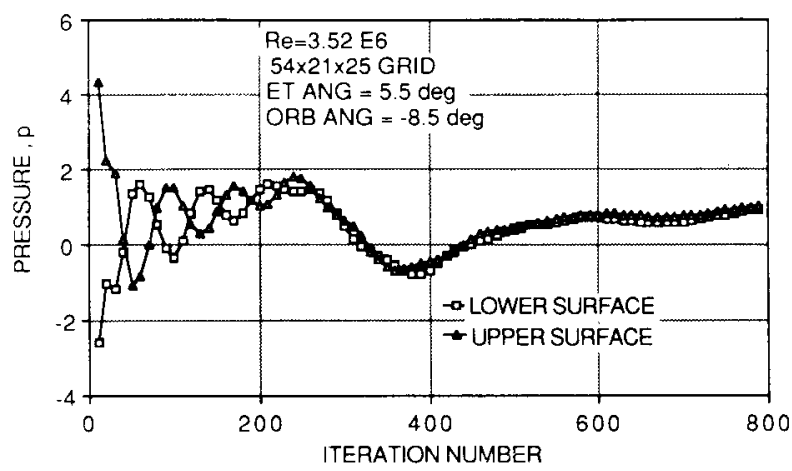

Fig. 4b Pressure history at the center of the Orbiter flapper.

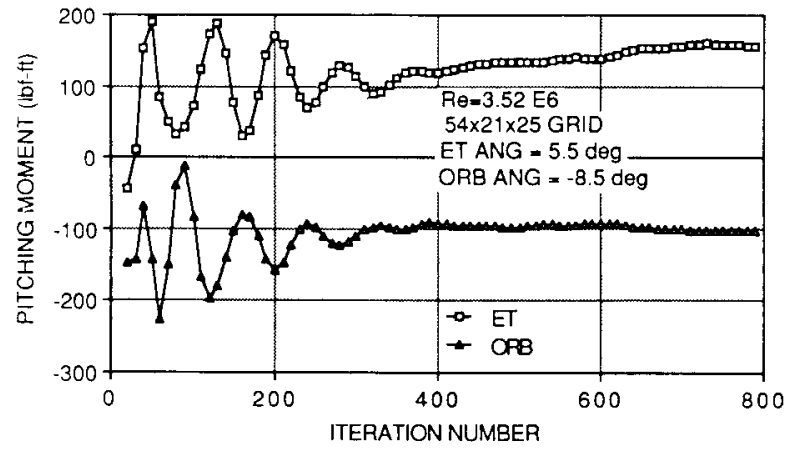

Fig. 5 Pitching moment history.

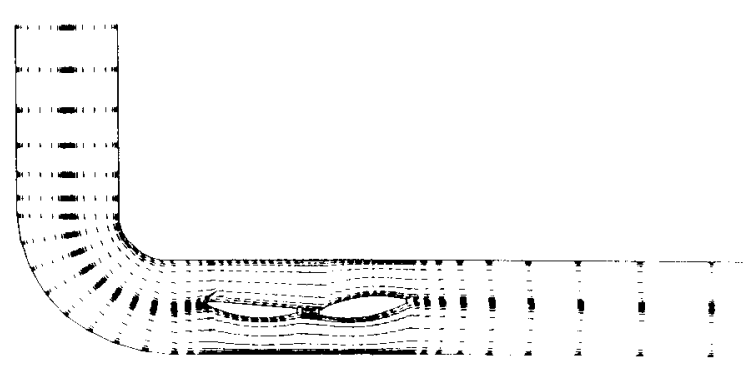

Fig. 6a Velocity vectors in the $x-z$ plane at $R e=3.5 \times 10^{6}$.

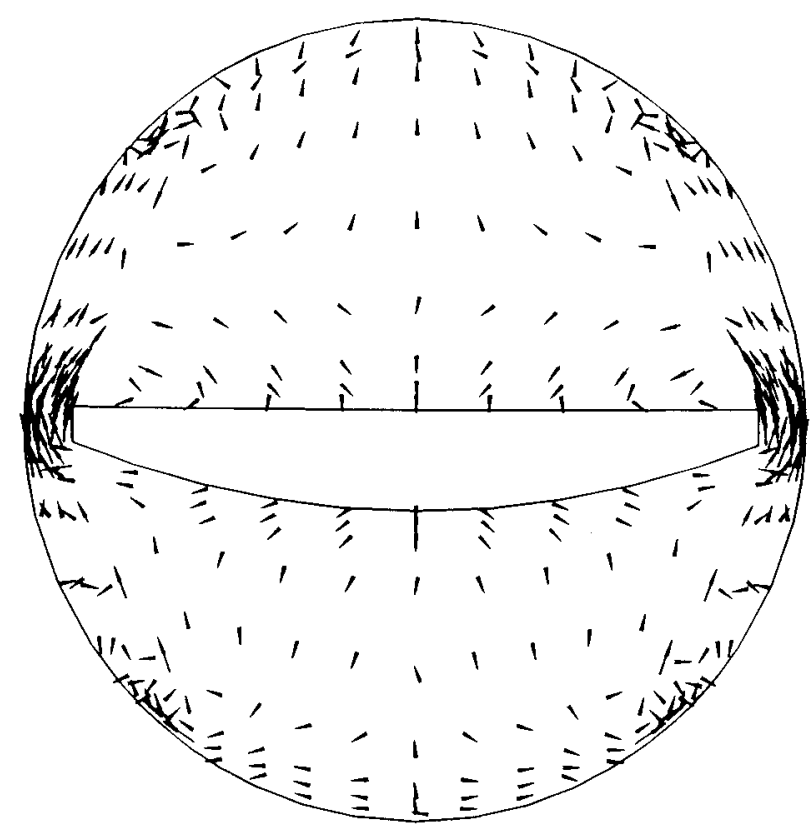

Fig. $6 \mathrm{~b}$ Velocity vectors in the flow cross-section at the center of the ET flapper at $R e=3.5 \times 10^{6}$.

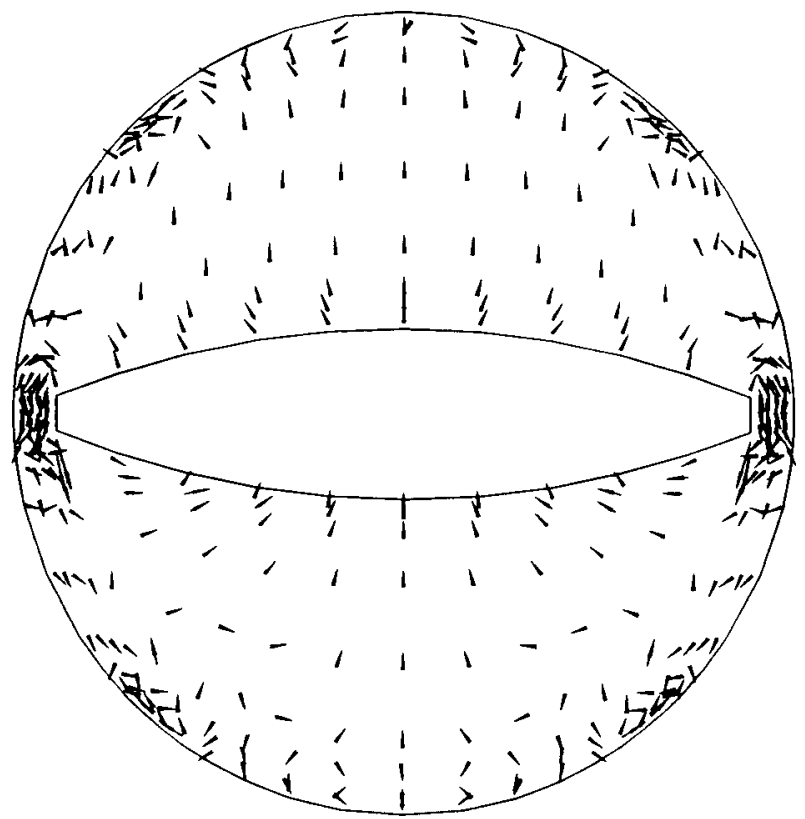

Fig. 6c Velocity vectors in the flow cross-section at the center of the Orbiter flapper at $R e=3.5 \times 10^{6}$. 


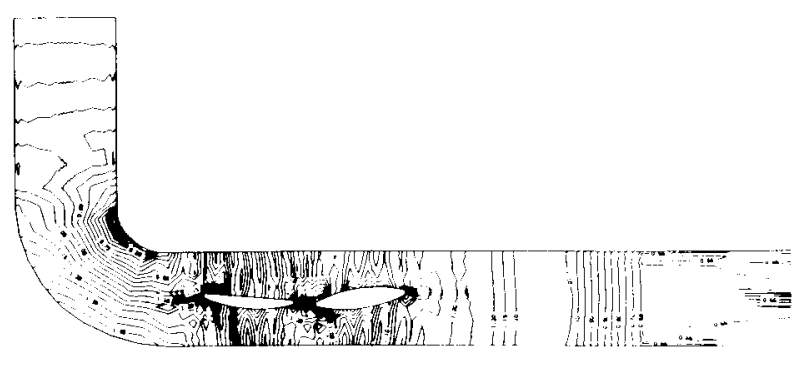

Fig. 7a Pressure contours in the $x-z$ plane at $R e=3.5 \times 10^{6}$.

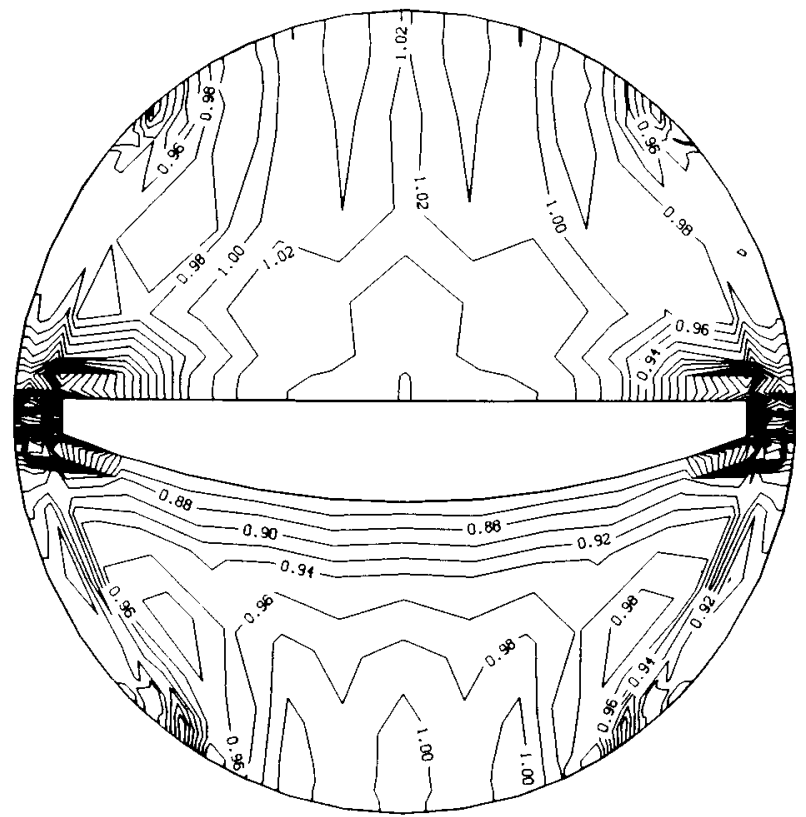

Fig. 7b Pressure contours in the flow cross-section at the center of the ET flapper at $R e=3.5 \times 10^{6}$.

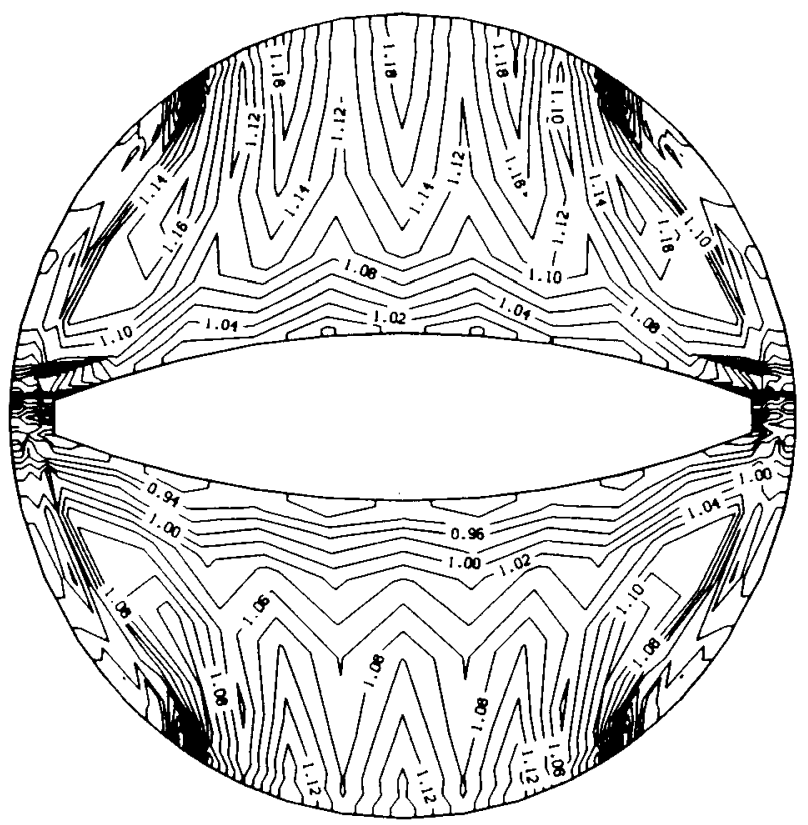

Fig. 7c Pressure contours in the flow cross-section at the center of the Orbiter flapper at $R e=3.5 \times 10^{6}$.

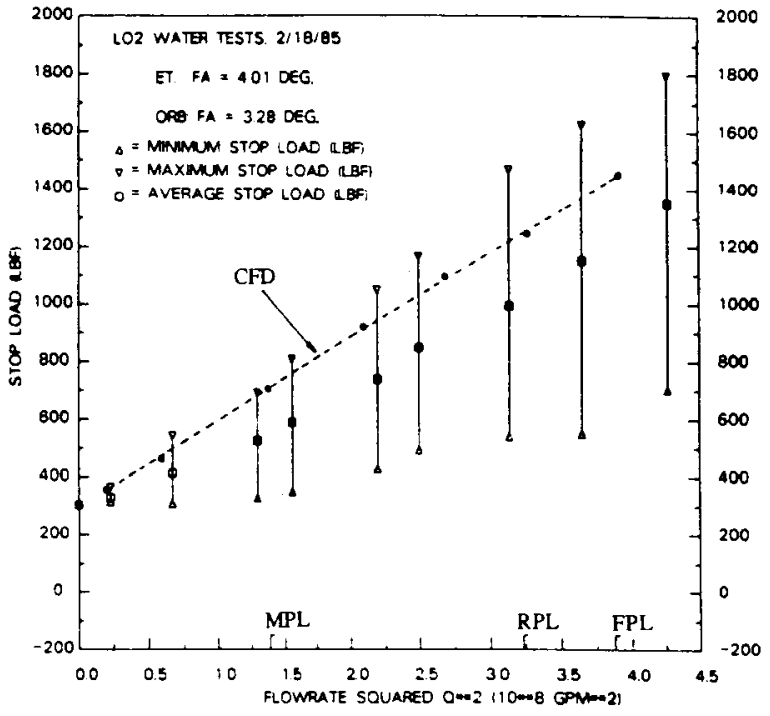

Fig. 8a Comparison of predicted stop load on the ET flapper with water flow data at various flow rates.

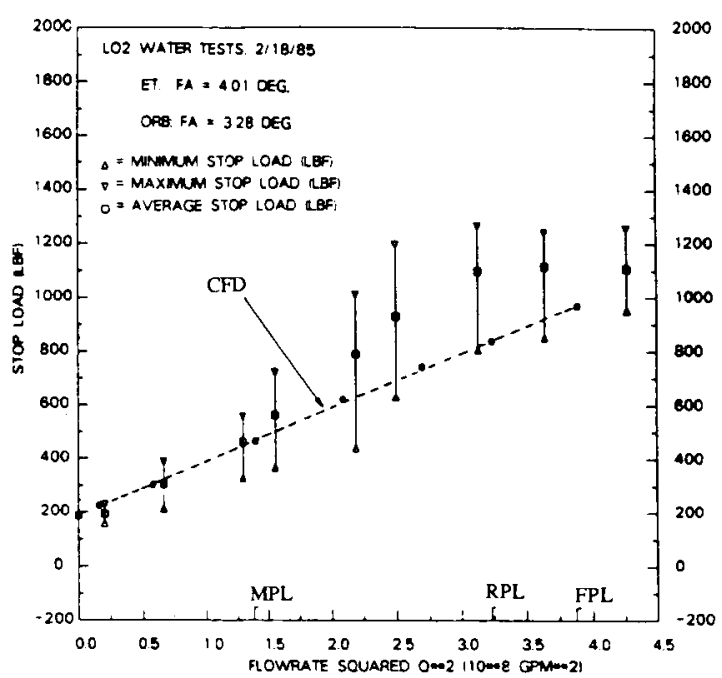

Fig. 8b Comparison of predicted stop load on the Orbiter flapper with water flow data at various flow rates.

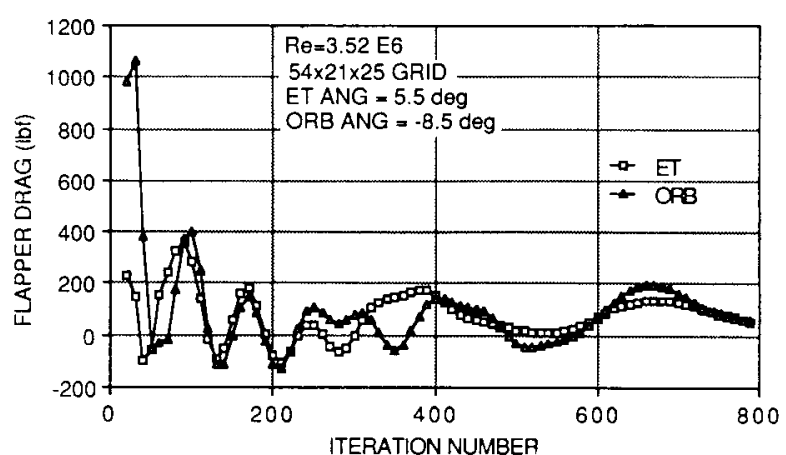

Fig. 9 Drag history for ET and Orbiter flappers. 